\title{
The Assessment of Intervention Programs for Autism Including Children and their Parents
}

\author{
Samirah Fahad Albalawi ${ }^{1 *}$ and Ahed Alkhatib ${ }^{2}$ \\ ${ }^{1}$ Alamal Complex for Mental Health Tabuk, Saudi Arabia \\ ${ }^{2}$ Department of Legal Medicine, Toxicology of Forensic Medicine, School of Medicine, Jordan University of Science and Technology, Jordan \\ *Corresponding author: Samirah Fahad Albalawi, Alamal Complex for Mental Health Tabuk, Saudi Arabia \\ To Cite This Article: Samirah Fahad A, Ahed Alkhatib. The Assessment of Intervention Programs for Autism Including Children and their \\ Parents. Am J Biomed Sci \& Res. 2019 - 1(6). AJBSR.MS.ID.000553. DOI: 10.34297/AJBSR.2019.01.000553
}

Received: March 07, 2019 | Published: March 13, 2019

\begin{abstract}
This is a review study regarding intervention programs for children with Autism Spectrum Disorder (ASD) from a family concept including both children with autism and their parents, because parents are considered essential parts for the successfulness of any intervention programs. ASD is considered to be a long-term neurodevelopmental disorder that is a long-standing problem in the context of social communication and interaction and the dominance of limited, repetitive patterns of behavior, interests or activities. Intervention programs aim to help ASD patients, especially in the early stages of development. Another objective is to involve the parents of children with ASD in order to improve the effectiveness of the implementation of these interventions. Reviewing the literature has shown that parental involvement reduces stress.
\end{abstract}

Keywords: Autism, autism spectrum disorders (asd); Neurodevelopment; Intervention; Social communication; Social interaction

\section{Introduction}

\section{An overview of the autism}

Autism spectrum disorder (ASD) is considered a long-term disorder of neurological development, which has long been associated with communication and social interaction, as well as with limited and repeated behavior, the domain of interests or activities [1,2]. ASD affects people in different ways, depending on the severity and possible coexistence with other diseases. In any case, the way the world understands, and acts is known. This is called "autistic culture" [3].

Of 68 children, one is going to have ASD (Center for Disease Control and Prevention, 2014) [4]. Children who have ASD usually exhibit important problems related to social and communication (American Psychiatric Association, 2013) [5]. These problems have been reported to be associated with behavioral challenges including self-injury, aggression, and severe noncompliance [6-8].

Several studies across the literature have shown that behavior problems have negative influences on the psychology of children as well as emotional and physical health [9]. The parents of children with ASD have been shown to be more stressed as compared to parents of normal developing children [10]. The study of Head \& Abbeduto [11] showed that stress of parents of ASD children impacts their ability to fully engage intervention programs which lowers the expectations of positive results for their children.

Several studies have reported that ASD symptoms can be reduced and the persons who have ASD have the ability to experience improved communication skills as well as adaptive behaviour [12-14].

\section{Targeting intervention programs of autistic children and their parents}

Although literature witnessed the existence of various intervention programs to improve people with ASD lifestyle [2], two main approaches have been reported of which the first is focused intervention practice to be used for a limited period of time, and Comprehensive Treatment Models (CTM) in which several interventional components are used for a long period of time to achieve a broader developmental impact [15].

Several studies showed that the access to teaching programs is impacted by factors such as sociodemographic and logistical factors, a matter that could limit the engagement of such early intervention programs [16-18]. It is worth mentioning that the earlier the engagement of intervention programs, the better the results due to the consideration that there is an advantage of the 
plasticity that usually occurs through early development [19]. Furthermore, early intervention participation may give predictions on acquiring of daily living skills that are essential for independent living [14].

Across literature, a variety of interventions programs have been described for children with ASD among which behaviourally based teaching was the most effective [12], particularly if parents were involved in these programs [20]. It has been realized through various reviews that the involvement of family as an essential element for effective treatment of children with ASD [12,21]. On this context, several behaviours were improved through the involvement of parent training such as children's joint attention, joint engagement, intellectual development, adaptive behaviour, compliance, and dyadic communication [14,22-26].

Pennefathera et al. [27] conducted a study in the light of considerations that the parents who had autism spectrum disorder (ASD) are likely to develop stress which may due to expression of problem behavior by their children. However, literature indicates that the implementation of intervention programs by parents, such as applied behavior analytic (ABA) lower their child's challenging behavior. Due to continuous expression of increasing stress rates by parents, it is expected that such stress may impact the implementation of such programs. According to the authors, the addition of other approaches including cognitive-affective strategies such as ACT and optimism training to ABA could benefit parents to further lower stress rates. Tele health models were reported to reach parents who are not able to attend parent training programs. The study included 23 parents of autism children with ages 4-8 years. The training program was a three-week online training program. Training program included different topics such as how to apply ABA principles, how to lower stress, and mediation practice. The results showed that the intervention program had the following advantages: decreased parental stress, increased knowledge about the autism, increased prosocial behavior, decreased hyperactive behaviors, and increased levels of satisfaction with the intervention.

Dai et al. [28] conducted a study in the light of considerations that behavioral intervention programs that target the involvement of parents to be an effective in lowering ASD, but the problem they are experiencing is their limited access. In this context, Dai et al purposed to investigate if a program based on video training of parents can be comprehensive and accepted to parents, can expand the knowledge of parents concerning strategies of behavior, and can increase parental self-efficacy. The study included 29 parents of children with ASD within the age 18-70 months. Parents completed the Early Intervention Parenting Self-Efficacy Scale [29]. Parents, who were included in the Treatment Group, were permitted to access to a parent-training (PT) program for teaching and behavior management techniques. It is worth to mention that the authors took into their account the culture of parents. Study findings showed that training program was accepted by parents and rated as valuable. The program increased self-efficacy and knowledge of parents on effective teaching strategies.

Nicole et al. [30] conducted a study to determine the effectiveness of JumpStart ${ }^{\mathrm{TM}}$, a 4-week educational program that includes a behavioral skills training model for parents with ASD. The goal of JumpStart is to teach parents how to behave in order to create a comprehensive intervention program. The study included 18 parents (treatment group) and a control group (n = 18). The variables of the study were changes in parents' selfefficacy, knowledge, stress, depression, ability to apply intervention programs, and ability to respond to a child. The results of the study showed a significant increase in the level of all variables between the treatment group and the control group.

\section{Conclusion}

ASD is a developmental disease that impacts communication and social interactions. The intervention programs at early stages are more effective in delivering effective treatments. The involvement of parents in intervention programs were shown to be beneficial for parents to reduce their stress, and for their children. Recent intervention programs have focused in the engagement of parents on video sessions. Early intervention programs have been used effectively to improve social communication.

\section{References}

1. American Psychiatric Association (2013) Diagnostic and statistical manual of mental disorders. Arlington ( $5^{\text {th }}$ Edn), American Psychiatric Publishing, USA.

2. Pilar Sanz-Cervera, M Inmaculada Fernández-Andrés, Gemma PastorCerezuela, Raúl Tárraga-Mínguez (2018) The effectiveness of teacch intervention in autism spectrum disorder: A review study. Papeles del Psicólogo 39(1): 40-50.

3. Mesibov GB, Shea V, Schopler E (2005) The TEACCH Approach to Autism Spectrum Disorders. New York: Academic/Plenum Publishers.

4. Center for Disease Control and Prevention (2014) Prevalence of autism spectrum disorders: Autism and developmental disabilities monitoring network, 11 sites United States, 2008. MMWR. Surveillance summaries: Morbidity and mortality weekly report. Surveillance summaries/CDC 63: $1-24$.

5. American Psychiatric Association (2013) Diagnostic and statistical manual of mental disorders. ( $\left.5^{\text {th }} \mathrm{Edn}\right)$, Washington DC, USA.

6. Baghdali A, Pascal C, Grisi S, Aussilloux C (2003) Risk factors for selfinjurious behaviors among 222 young children with autistic disorders. Journal of Disability Research 47(8): 622-627.

7. Hartley S L, Sikora DM, McCoy R (2008) Prevalence and risk factors of maladaptive behaviour in young children with autistic disorder. Journal of Intellectual Disability Research 52(10): 819-829.

8. Kanne SM, Mazurek MO (2011) Aggression in children and adolescents with ASD: prevalence and risk factors. Journal of Autism and Developmental Disorders 41(7): 926-937.

9. Kuhlthau KA, Bloom S, Van Cleave J, Knapp AA, Room D, et al. (2011) Evidence for family-centered care for children with special health care needs: A systematic review. Academic Pediatrics 11(2): 136-143.

10. Schieve LA, Blumberg SJ, Rice C, Visser SN, Boyle C (2007) The relationship between autism and parenting stress. Pediatics 119(1): 114-121.

11. Head LS, Abbeduto L (2007) Recognizing the role of parents in developmental outcomes: A systems approach to evaluating the child with developmental disabilities. Mental Retardation and Developmental Disabilities Research Reviews 13(4): 293-301.

12. Rogers SJ, Vismara LA (2008) Evidence-based comprehensive treatments for early autism. Journal of Clinical Child and Adolescent Psychology 37(1): 8-38. 
13. Anderson DK, Liang JW, Lord C (2014) Predicting young adult outcome among more and less cognitively able individuals with autism spectrum disorders. Journal of Child Psychology and Psychiatry 55(5): 485-494.

14. Bal VH, Kim S, Cheong D, Lord C (2015) Daily living skills in individuals with autism spectrum disorder from 2 to 21 years of age. Autism 19(7): 774-784.

15. Odom SL, Boyd BA, Hall LJ, Hume K (2010) Evaluation of comprehensive treatment models for individuals with autism spectrum disorders. Journal of Autism and Developmental Disorders 40(4): 425-436.

16. Sharpe DL, Baker DL (2007) Financial issues associated with having a child with autism. Journal of Family and Economic Issues 28(2): 247264.

17. Thomas KC, Ellis AR, McLaurin C, Daniels J, Morrissey JP (2007) Access to care for autism-related services. Journal of Autism and Developmental Disorders 37(10): 1902-1912.

18. Chiri G, Warfield ME (2012) Unmet need and problems accessing core health care services for children with autism spectrum disorder. Maternal and Child Health Journal 16(5): 1081-1091.

19. Dawson G (2008) Early behavioral intervention, brain plasticity, and the prevention of autism spectrum disorder. Development and Psychopathology 20(3): 775-803.

20. Tonge B, Brereton A, Kiomall M, Mackinnon A, Rinehart NJ (2014) A randomised group comparison-controlled trial of 'preschoolers with autism': A parent education and skills training intervention for young children with autistic disorder. Autism 18(2): 166-177.

21. Hurtb J, Shaw E, Izeman SG, Whaley K, Rogers S J, et al. (1999) Areas of agreement about effective practices among programs serving young children with autism spectrum disorders. Infants and Young Children 12(2): 17-26

22. Bahadourian AJ, Greer RD (2005) CABAS parent education: Increasing child compliance via parental emission of unflawed commands and contingent consequations during play. Journal of Early and Intensive Behavior Intervention 2(3): 213-222.

23. Rocha ML, Schreibman L, Stahmer AC (2007) Effectiveness of training parents to teach joint attention in children with autism. Journal of Early Intervention 29(2): 154-172.

24. Green J, Charman T, McConachie H, Aldred C, Slonims V, et al. (2010) Parent-mediated communication-focused treatment in children with autism (PACT): A randomized controlled trial. The Lancet 375(9732): 2152-2160.

25. Vismara L A, Young GS, Rogers SJ (2012) Telehealth for expanding the reach of early autism training to parents. Autism Research and Treatment 121878: 12

26. Kasari C, Gulsrud A, Paparella T, Hellemann G, Berry K (2015) Randomized comparative efficacy study of parent-mediated interventions for toddlers with autism. Journal of Consulting and Clinical Psychology 83(3): 554-563.

27. Jordan Pennefathera, Meme Hienemanb, Tracy J Raulstona, Nell Carawaya (2018) Evaluation of an online training program to improve family routines, parental well-being, and the behavior of children with autism. Research in Autism Spectrum Disorders 54: 21-26.

28. Yael G Dai, Lynn Brennan, Ariel Como, Jamie Hughes-Lika, Thyde Dumont-Mathieu, et al. (2018) A video parent-training program for families of children with autism spectrum disorder in Albania. Research in Autism Spectrum Disorders 56: 36-49.

29. Guimond AB, Wilcox MJ, Lamorey SG (2008) The Early Intervention Parenting Self-Efficacy Scale (EIPSES) scale construction and initial psychometric evidence. Journal of Early Intervention 30(4): 295-320.

30. Nicole L Matthews, Beatriz C Orr, Brittani Harris, Rachel McIntosh, Daniel Openden, et al. (2018) Parent and child outcomes of JumpStart ${ }^{\mathrm{T} M}$ an education. 